\title{
MR ANGIOGRAPHY ACCELERATION TECHNIQUES WITH ITERATIVE RECONSTRUCTIONS: THE IT-TWIST SEQUENCE
}

\author{
Curatolo Calogero
}

Dott. TSRM Ordine PSTRP TSRM Agrigento-Caltanissetta

KEYWORDS: RM, Angio-RM, Iterative reconstruction

\section{ABSTRACT}

In recent years MR Angiography allowed to assess blood vessels and flow in a non-invasive way. The aim of our work was to illunstrate a modern MR Angiography technique, the $4 D$ Iterative reconstruction Time resolved angiography With Interleaved Stochastic Trajectories (IT-TWIST), capable to generate angiograms with high spatial and temporal resolution, providing multiple and continuous post-contrastographic sets of images. Technological and physical characteristics are reported in detail and consequent advantages are summarized.

\section{IINTRODUCTION}

MR Angiography is an imaging method that allows an accurate and non-invasive assessment of blood flow and blood vessel morphology. The sequences used in recent years have undergone considerable modifications with the aim of improving not only the temporal resolution, which is fundamental for angiographic imaging in MRI, but also to give a significant response in terms of spatial resolution.

\section{PURPOSE}

MR angiography techniques are increasingly required to replace or in addiction to the conventional methods used until now. The aim of this study is to present the new method 4D Iterative reconstruction Time resolved angiography With Interleaved Stochastic Trajectories (IT-TWIST) designed to provide angiograms with high spatial resolution by applying algorithms that allow to obtain multiple and continuous sets of images.

\section{MATERIAL AND METHODS}

After an analysis of data published in literature, technological and physical characteristics, and consequent advantages of the Angio-RM 4D Time Resolved technique and its most recent combination with iterative reconstructions, are reported and summarized in detail.

\section{RESULTS AND DISCUSSION}

Among the various techniques applied in MRAngiography the 4D Time Resolved are currently those that provide static images with high quality and high temporal resolution, able to document the dynamism of post-contrast phases

The Iterative reconstruction Time resolved angiography With Interleaved Stochastic Trajectories It-TWIST is a sequence that combines parallel imaging techniques and efficient subsampling methods with the iterative data reconstruction system.
The technology is based on a sequence Rapid Acquisition GRE-Pulse Fid-Imaging Spoiled T1w with very short TR (2-3ms), short TE (1-2 ms) and an FA ranging between 15-25- compete with a broad bandwidth and a view-sharing sampling combined with "Key-hole" method in which the K-space is divided into two parts: a central region $\mathrm{A}$ and a peripheral region $\mathrm{B}$, the latter divided into random sub-regions "b1-b2-b3..."

After contrast medium administration, the images are acquired in several consecutive stages. In particular, the data of central region A, that carry informations about the contrast of the image, are acquired completely and continuously, in association with the data of sub-regions " $b$ " which are alternatively and stochastically acquired at each stage, and which carry informations related to spatial resolution.

Iterative Reconstruction is then applied to each cycle, i.e., a collection of region A data is created together with the raw data iteratively reconstructed in subregion " $b$ " and data-matching of the other sub-regions $\mathrm{b}$, stochastically obtained in the various stages. This operation continues until the ideal condition obtained through a comprehensive research of parameters in the space is met, thus applying a data loop on several steps, refining and improving the result thanks to a cost function that guides the mathematical relationship with its own ratio accuracy/ speed of search.

Although in MR Imaging a compromise between Time Resolution and image quality exists, IT-TWIST seems to resolve both conditions. The implementation of Iterative Reconstruction applied to the sequence 4D Time resolved angiography With Interleaved Stochastic Trajectories effectively contributes to the "denoising" of images thanks to the greater robustness provided by the convolution of data, providing a higher degree of images quality than conventional Contrast Enhanced MR-Angiography and an optimal contrast-enhancement intensity between the various phases, with a finer and detailed delineation of the main artero-venous and secondary branches. 


\section{REFERENCES}

1. Winterer J. T. , Blanke P., Schaefer A. , Pache G. , Langer M. , Markl M. Bilat- eral contrast-enhanced MR angiography of the hand: Diagnostic image quality of accelerated MRI using echo sharing with interleaved stochas- tic trajectories (TWIST). Eur Radiol 2011;21:1026-1033.

2. Vogt F. M. , Theysohn J. M. , Michna D. , et al. Contrast-enhanced time-resolved 4D MRA of congenital heart and vessel anomalies: Image quality and diagnostic value compared with 3D MRA. Eur Radiol 2013; 23:23922404.

3. Stalder A. F., Schmidt M., Quick H. H. , et al. Highly undersampled contrast- enhanced MRA with iterative reconstruction: Integration in a clinical setting. Magn Reson Med 2015;74:1652-1660.

4. Wetzl J. , Forman C. , Wintersperger B. J., et al. High-resolution dynamic CE-MRA of the thorax enabled by iterative TWIST reconstruction. Magn Reson Med 2017;77:833-840.

5. Yokota Y. , Fushimi Y. ,Okada T. , Fujimoto K. , Oshima S. , Nakajima S. , Fujii T. , Tanji M. , Inagaki N. , Miyamoto S. , Togashi K. , Evaluation of Image Quality of Pituitary Dynamic Contrast-Enhanced MRI Using Time- Resolved Angiography With Interleaved Stochastic Trajectories (TWIST) and Iterative Reconstruction TWIST (IT-TWIST), j. magn. reson. imaging 2019. doi: 10.1002/jmri.26962

6. Laub G. , Kroeker R. , syngo TWIST for dynamic time-resolved MR angiography. MAGNETOM Flash 2006; 3;9295. (Brochure from Siemens explaining TWIST).

7. Hennig J. ,Scheffler K. ,Laubenberger J. ,Strecker R. Time-resolved projection angiography after bolus injection of contrast agent. Magn Reson Med 1997; 3:341-345. (Basis of TWIST)

8. Rapacchi S. , Natsuaki Y. , Plotnik A. , et al. Reducing view-sharing using compressed sensing in time-resolved contrast-enhanced magnetic res- onance angiography. Magn Reson Med 2015;74:474-481. 